\title{
Equidade: seus conceitos, significações $e$ implicações para o SUS
}

\section{Equity: concepts, meanings and implications for the Brazilian National Health System}

\author{
Fernando Passos Cupertino de Barros \\ Universidade de Brasília. Faculdade de Ciências da Saúde. \\ Goiânia, GO, Brasil. \\ E-mail: fernandocupertinoœgmail.com

\section{Maria Fátima de Sousa} \\ Universidade de Brasília. Faculdade de Ciências da Saúde \\ Brasília, DF, Brasil. \\ E-mail: fatimasousa®unb.br
}

\section{Resumo}

Este ensaio tem por objetivo rever os diferentes elementos conceituais de equidade que foram se consolidando ao longo de tempo e a leitura que hoje se faz no Brasil de sua significação, orientada como um caminho para que se possa atingir um nível de garantia real e de fruição plena dos direitos à saúde, tendo-se sempre em mente a preocupação em compreender as diferenças, as necessidades e as particularidades dos distintos grupos sociais. Destaca a necessidade de se pensar a equidade em saúde como um processo em permanente transformação, que vai mudando seu escopo e abrangência à medida que certos resultados são alcançados e que novos desafios vão surgindo. Propõe que se deve avançar na discussão do tema, de modo a se estabelecer balizas seguras que resultem em práticas de promoção da equidade necessária à concretização do direito à saúde, capazes de produzir a melhoria objetiva de indicadores de saúde, bem como da percepção do cidadão com relação à satisfação com seu próprio estado de saúde, o de sua família e o da comunidade a que pertence. Assim sendo, o ideal de um sistema de saúde capaz de garantir o necessário a todos, levando-se em conta suas singularidades e necessidades, poderá ser atingido.

Palavras-chave: Equidade; Equidade em Saúde; Sistema Único de Saúde.

\section{Correspondência}

Rua 7, 201, apto 202. Setor Oeste, Goiânia, GO, Brasil. CEP $74110-090$ 


\section{Abstract}

This paper aims to review the different conceptual elements of equity that have been consolidated over time and the reading of its signification that is made in Brazil today, conceived as a way to achieve the guarantee and full advantage of rights to health, bearing in mind the concern to understand the differences, needs and particularities of different social groups. We emphasize the need to conceive equity in health as a process in permanent transformation, which keeps changing its scope and comprehensiveness as results are achieved and new challenges occur. We propose to advance in the discussion of the topic to establish solid checkpoints that result in promotion of the equity needed to achieve the right to health, allowing the production of an objective improvement of health indicators, as well as of the citizen perception of his satisfaction concerning his/her health condition and of his/ her family and community. Therefore, the ideal of a health system capable of providing what is necessary for everybody, taking into account specificities and particular needs, can be achieved.

Keywords: Equity; Equity in Health; Brazilian Health System.

\section{Introdução}

Ao se considerar a política de saúde como uma política social, uma implicação decorrente é a de que a saúde é um dos direitos inerentes à condição de cidadania, uma vez que a participação plena dos indivíduos na sociedade política somente se realiza a partir de sua inserção como cidadãos (Fleury; Ouverney, 2008). Mais que um direito social, há uma tendência cada vez mais perceptível de se considerar o direito à saúde como um direito humano (Garrafa; Oselka; Diniz, 2014). Em 200o, o Comitê dos Direitos Econômicos, Sociais e Culturais das Nações Unidas aprovou o Comentário-Geral $\mathrm{N}^{\circ} 14$, que reafirmou a saúde como direito humano fundamental. 0 texto enfatiza que "o direito à saúde não é para ser entendido como o direito de ser saudável”, mas como um direito inclusivo ampliado não somente ao acesso aos cuidados médicos apropriados, mas também a determinantes de saúde como o acesso à água potável e saneamento adequado, segurança alimentar, nutrição e moradia, condições ocupacionais e ambientais saudáveis e acesso à informação e educação em saúde, incluindo saúde sexual e reprodutiva (ONU, 2000).

O documento mencionado destaca o papel importante da participação comunitária “nas decisões relacionadas à saúde, nos níveis comunitários, nacionais e internacionais". 0 texto ressalta a não discriminação, a disponibilidade, a acessibilidade (física, econômica e de informações), a aceitabilidade (incluindo as questões éticas e culturais apropriadas) e a qualidade dos serviços (incluindo qualidade médica e científica) (Jillson; Eisenstein, 2013).

Na América Latina, a partir de meados dos anos 1970, tem início no México e no Equador, e, posteriormente, no Brasil, um movimento do setor acadêmico que procurou recuperar as ideias de causalidade social do processo saúde-doença que haviam declinado após o advento da era bacteriológica. No Brasil, esse movimento, intitulado Movimento da Reforma Sanitária, foi protagonizado por Arouca, com suas críticas ao preventivismo, e por Donnangelo, com seus estudos sobre saúde e sociedade (Silva; Barros, 2002). Não se pode, ainda, esquecer o papel propulsor exercido pelos postulados da Conferência 
de Alma-Ata, em 1978, com sua ambição de "saúde para todos no ano 2000", cujo documento final salienta a interferência da desigualdade social nas políticas de saúde, ressaltando o papel que a lacuna entre países desenvolvidos e em desenvolvimento então representava, exortando ainda todos os países à cooperação, na busca pelo objetivo comum da saúde em uma ação entendida como direito e dever de todos, individual e coletivamente (Brasil, 2002).

A Constituição Federal Brasileira de 1988 reconheceu e deu força constitucional a princípios e pensamentos defendidos pelo Movimento da Reforma Sanitária. Assim, valores sociais como o direito de acesso universal e a integralidade da atenção passaram a refletir a opção da sociedade por um sistema de saúde típico de um Estado de bem-estar, em substituição ao sistema excludente e desigual que existia antes de 1988. Entretanto, a equidade, ainda que citada com frequência entre os princípios ou diretrizes do SUS, a rigor, não tem explicitamente a mesma estatura jurídica dos demais (universalidade, integralidade, descentralização, participação social) (Piola et al, 2009). Apesar disso, cada vez mais a preocupação com a equidade encontra-se presente no pensamento sanitário brasileiro. Nos relatórios das conferências nacionais de saúde de 1992, 1996 e 200o, por exemplo, a utilização do termo "equidade", ou equivalente, é crescente: no

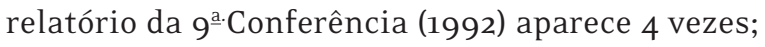
no da 10 ${ }^{\underline{a}}$ Conferência (1996), 14 vezes e 32 vezes no da 11 ${ }^{\mathrm{a}}$-Conferência (200o) (Pinheiro; Westphal; Akerman, 2005). Já no documento orientador para os debates da 14 ${ }^{\text {a. }}$ Conferência (2011), a preocupação com a equidade é o primeiro dos cinco tópicos propostos e intitula-se "Avanços e desafios para a garantia do acesso e do acolhimento com qualidade e equidade"(Brasil, 2011).

Este ensaio tem como objetivo rever os diferentes elementos conceituais de equidade que foram se consolidando ao longo de tempo, e a leitura que hoje se faz no Brasil de sua significação, orientada como um caminho para que se possa atingir um nível de garantia real e de fruição plena dos direitos à saúde, tendo sempre em mente a preocupação em compreender as diferenças, as necessidades e as particularidades dos distintos grupos sociais. Afi- nal, há que se pensar a equidade em saúde como um processo em permanente transformação (Escorel, 2008) que vai mudando seu escopo e abrangência à medida que certos resultados são alcançados e que novos desafios vão surgindo.

\section{Considerações de ordem conceitual}

Arendt lembra-nos de que a cidadania, sendo o "direito a ter direitos", é geradora de um espaço público de igualdade entre os indivíduos tornados cidadãos (Arendt, 1987). Por outro lado, os direitos sociais, como o direito à saúde, estabelecem um marco de ampliação da cidadania nas sociedades modernas, visto que, ao contrário dos direitos individuais civis e políticos, exigem a intervenção do Estado e incorporam novos princípios ao desenvolvimento de padrões de cidadania, em particular formas diferenciadas de compreender o significado de "satisfazer um nível decente de bem-estar e seguridade econômica e social”. O princípio de igualdade que orienta a cidadania desdobra-se no princípio da universalidade das regras de distribuição (princípios universalistas de justiça). Esse ideal de universalidade tende à homogeneização, como parte da vontade geral, e acaba por diluir as diferenças, o que definitivamente prejudica grupos de cidadãos menos favorecidos.

A introdução do princípio da equidade - entendida como um elemento de diferença dentro do espaço da cidadania, que é um espaço de igualdade - é acompanhada no âmbito dos direitos pelo desenvolvimento de uma terceira geração, a dos direitos difusos e coletivos, que se distinguem dos que integram a chamada primeira geração (os direitos individuais) e a segunda geração (os direitos sociais e econômicos). Tal distinção, em razão de sua coletividade e de seu aspecto difuso, termina por introduzir princípios de solidariedade, tolerância e confiança. São difusos porque sua titularidade não é clara, englobando tanto interesses públicos quanto privados, e correspondem às necessidades comuns a conjuntos de indivíduos e que somente podem ser satisfeitas a partir de uma ótica comunitária (Escorel, 2001).

Equidade tem sido uma expressão bastante utilizada quando tratamos de direitos sociais, tan- 
to em sentido mais genérico quanto em um mais específico. No primeiro, termina por confundir-se com o próprio sentido de justiça na perspectiva do estabelecimento de regras justas para a vida em sociedade, o que a aproxima do conceito e do sentido de igualdade, frequentemente assim entendidos no âmbito da saúde (Almeida, 2002; Campos, 2006; Starfield, 2001). Já no segundo, para diferentes pensadores - de Aristóteles a Norberto Bobbio -, a equidade é uma adaptação da norma geral a situações específicas, pois a aplicação de uma norma genérica quando empregada literalmente, sem se levar em conta as especificidades de diferentes situações, poderia produzir injustiça. Assim, essa concepção de equidade como julgamento e intervenção situacional, isto é, de acordo com cada caso, pressupõe a inexistência de estrutura, normas ou sistemas de leis ou conhecimentos perfeitos (Campos, 20o6). A equidade poderia então ser uma prática indispensável para se chegar ao ideal de justiça e de cidadania plena, capazes de garantir o gozo de uma situação de igual bem-estar para todos os cidadãos.

Na vida em sociedade, sobretudo no modo capitalista, coexistem situações de abundância e de miséria, o que determina a existência de desigualdades absolutas e relativas, as primeiras ligadas à significativa diferença de renda entre pessoas ou entre grupos sociais; a segunda, associando a essa dimensão as demais condições de vida. O recorte mais adequado ao exame das desigualdades sociais parece relacionar-se com a identificação de situações que envolvem algum grau de injustiça, uma vez que colocam determinados coletivos populacionais em desvantagem quanto à chance de serem e de se manterem sadios (Bagrichevsky et al., 2013). Tal problemática reflete muitas vezes os níveis de segregação existentes, que decorrem, por sua vez, da forma de organização e do modo de produção da sociedade, configurando-se como iniquidade. Contudo, no âmbito da formulação de políticas, o significado puramente semântico para o termo "desigualdade" nem sempre corresponde a algo prejudicial. Assim, o conceito de iniquidade ganhou forma, firmando-se como sinônimo de toda e qualquer "desigualdade injusta" a ser permanentemente evitada ou combatida (Almeida, 2002). Nesse aspecto, Whitehead é, possivelmente, a autora mais citada na literatura internacional que trata das questões conceituais de equidade em saúde (Vieira-da-Silva; Almeida Filho, 2009). Para ela, iniquidades constituem diferenças que, além de evitáveis, são também injustas (Whitehead, 1992).

Segundo Sen (2002), a equidade em saúde tem múltiplas facetas, e o melhor é vê-la como um conceito multidimensional que inclui aspectos relacionados ao nível de saúde que se tem e à possibilidade de se obtê-la, e não apenas com a distribuição do cuidado sanitário. Inclui a justiça dos processos e, por conseguinte, deve-se atentar para a ausência de discriminações na prestação da assistência. Ademais, um bom compromisso com a equidade em saúde necessita, também, que as considerações sobre a saúde sejam integradas a temas mais amplos de justiça social e de equidade global, com especial atenção sobre a versatilidade dos recursos e as diferenças de alcance e de impacto dos diferentes acordos sociais. Nesse sentido, Breilh (2010), ao correlacionar equidade e classe social, distingue equidade/iniquidade de igualdade/desigualdade ao afirmar que a iniquidade é a categoria que define as relações e contrastes de poder existentes numa formação social e resulta da acumulação histórica de poder, decorrente do processo no qual uma determinada classe social apropria-se das condições de poder que, em geral, são interdependentes: apropriação e acumulação econômica, política e cultural. Já desigualdade seria a expressão resultante dessa iniquidade, que expressa uma injustiça na repartição de bens e serviços que existem numa sociedade. Em outras palavras, enquanto a iniquidade é uma categoria explicativa, a desigualdade é uma expressão explícita da iniquidade.

Escorel, por sua vez, afirma que o princípio da equidade surgiu no período contemporâneo associado aos direitos das minorias, introduzindo a diferença no espaço público da cidadania, que tem por princípio basilar a igualdade. 0 reconhecimento da diferença entra em conflito com o pensamento jurídico clássico, que percebe a cidadania como sendo comum e indiferenciada. Entretanto, dada a diversidade das sociedades modernas, a noção de igualdade só se completa se compartilhada com a de 
equidade, posto que não basta a existência de um padrão universal se não comportar o direito à diferença. O padrão passa, então, de homogêneo a equânime. Dessa forma, a ideia de equidade foi incorporada e até mesmo substituiu o conceito de igualdade, que significaria a distribuição homogênea: a cada pessoa uma mesma quantidade de bens ou serviços. Equidade, por sua vez, levaria em consideração que as pessoas são diferentes e que têm necessidades diversas (Escorel apud Pereira; Lima, 2008).

\section{A busca da equidade no âmbito do Sistema Único de Saúde}

No Brasil, tem-se procurado enfaticamente no campo da Saúde Coletiva destacar que a superação de desigualdades em saúde exige a formulação de políticas públicas equânimes, o que implica, por um lado, reconhecer a saúde como um direito de cidadania e, por outro, priorizar as necessidades como categoria essencial para a promoção de justiça. É preciso, ainda, incorporar à pauta certas diferenças e diversidades como as relacionadas à questão das condutas de risco e das heterogeneidades de base étnico-culturais (Vieira-da-Silva; Almeida Filho, 2009).

Seria ingenuidade supor que o SUS não tem desigualdades, as quais, de uma forma ou outra, persistem mesmo em países com sistemas universais bem mais antigos e consolidados do que o brasileiro, embora em escala bem mais reduzida do que em países onde prevaleça o mercado na saúde. Com o SUS, que institucionalizou o acesso universal e igualitário aos serviços de saúde, a exclusão formal desapareceu, mas não a iniquidade que subsiste em decorrência de fatores como a desinformação, associada aos diferenciais de escolaridade, ou ainda da deformação em determinadas políticas públicas, em algumas das quais ainda estão presentes os privilégios e a discriminação (Piola, 2009; Nunes et al., 2001)

\section{A tributação e o financiamento público da saúde}

A existência de financiamento suficiente, capaz de garantir acesso universal e integralidade dos cuidados de saúde à população é condição funda- mental para que tudo o mais possa acontecer. Assim, se levarmos em conta o comportamento do gasto em saúde na América Latina, não é difícil concluir que há uma patente incapacidade de assegurar coberturas universais, sendo os recursos altamente dependentes de regimes contributivos, ao que se soma a vulnerabilidade dessa política em relação ao comportamento pró-cíclico do gasto público, além de um elevado gasto privado (Fleury, 2011). O balanço das décadas de 1980 e de 1990 na América Latina, por exemplo, é desanimador, pois nele estão presentes um crescimento econômico inexistente ou muito modesto e cíclico; uma concentração de renda cada vez mais intensa e perversa; um importante aumento dos níveis de desigualdade, do número de pobres e da exclusão social, com crescentes limitações ao desenvolvimento e à superação dessas condições de pobreza (Almeida, 2002).

No Brasil, em particular, houve - e continua a existir, concomitantemente - alguns fatores que contribuem para manter e até mesmo aprofundar situações de iniquidades sociais, com seus inevitáveis reflexos sobre a saúde. Além de um gasto público que está significativamente em desacordo com o próprio sentido de existir de um sistema público de saúde, o país adota um sistema tributário altamente regressivo, que privilegia a taxação sobre o consumo em lugar daquela sobre renda e riqueza - o que acentua as desigualdades antes mesmo da alocação dos recursos, como bem lembra Fleury (2011). Portanto, antes de se gritar pelo necessário aumento no gasto público em saúde, é preciso perguntar quem efetivamente contribui para o orçamento público.

Agrava tal situação o fato de que a pretendida descentralização da saúde, ancorada em marcos legais, não se tem feito acompanhar por equivalente contrapartida financeira dos três entes federados. Entre 2000 e 2011, os estados e municípios ultrapassavam o triplo do volume de recursos destinados à saúde, passando de R\$ 28 bilhões para R\$ 89 bilhões, o que correspondeu a um incremento de R\$ 61 bilhões (sendo R\$ 28 bilhões referentes ao incremento estadual e R\$ 32 bilhões, ao municipal) (Conselho Nacional de Secretários de Saúde, 2011). Nesse mesmo período, a União aumentou o gasto em ações e serviços públicos de saúde em R\$ 31 
bilhões, que correspondeu a um aumento de $75 \%$ em relação a 200o. Esse valor incremental é muito próximo ao observado em cada uma das outras duas esferas de governo, totalizando um aumento da ordem de R\$ 92,7 bilhões. Assim, dois terços do aumento dos recursos para as ações e serviços públicos de saúde após a promulgação da Emenda Constitucional $n^{0} 29$ foram provenientes das receitas próprias de estados e municípios, enquanto um terço foi proveniente dos recursos injetados pela União. Contudo, mesmo com esses aumentos, o Instituto Brasileiro de Geografia de Estatística (IBGE) constatou que em 2009 o gasto público em saúde encontrava-se em torno de 3,8\% do Produto Interno Bruto (Oliveira, 2012), percentual muito inferior àquele aplicado por outros países que possuem sistemas universais de saúde (Piola et al., 2013).

O exame dos dados de 2013 da Organização Mundial da Saúde (OMS) sobre financiamento dos sistemas de saúde mostra que o Brasil gasta em saúde cerca de $9 \%$ do PIB. O valor indica que o Brasil tem um gasto total em saúde muito adequado, bem próximo à média dos gastos em saúde dos países desenvolvidos. Contudo, quando se examina o percentual do gasto público em saúde, verifica-se que é muito baixo e incapaz de garantir que a norma constitucional se materialize na prática social, de modo a cumprir o princípio da universalidade do SUS.

Os gastos públicos em saúde em nosso país são muito baixos quando comparados aos de outros países em dólares americanos com paridade de poder de compra. O gasto total em saúde é de 1.009 dólares, mas o gasto público per capita em saúde é de apenas 474. Esse valor é muito inferior aos valores praticados em países desenvolvidos e em países da América Latina como Argentina (US\$ 851,oo), Chile (US\$ 62,0o), Costa Rica (US\$ 825,00), Panamá (US\$ 853,00) e Uruguai (US\$ 740,oo). A razão para esse baixo gasto público em saúde no Brasil está no fato de que os gastos em saúde correspondem a 10,7\% dos gastos do orçamento total dos governos, um valor muito abaixo do praticado em âmbito internacional, em países desenvolvidos e em desenvolvimento (Mendes, 2013).

É comum a percepção de que os constituintes não previram as fontes dos recursos para implantar as políticas sociais, mas, no caso da saúde, ainda que não tenha sido possível solução duradoura, o art. 55 do Ato das Disposições Constitucionais Transitórias estabeleceu que um mínimo de $30 \%$ do orçamento da Seguridade Social, excluído o seguro-desemprego, seria destinado ao setor de saúde até a aprovação da Lei de Diretrizes Orçamentárias (LDO) (Gomes, 2014). Segundo Carvalho (2008), desde 1989 essas leis mantiveram a definição dos 30\% dos recursos da seguridade para a saúde, mas em 1994 o então Presidente da República, Itamar Franco, vetou esse dispositivo. Caso tivesse sido adotado como solução permanente, o nível federal disporia atualmente de mais que o dobro do orçamento.

Estudos demonstram que se a materialidade da política de saúde no Brasil pode ser vista a partir do comportamento dos gastos em saúde, observou-se um alinhamento de política, no que se refere ao financiamento, nos governos FHC e Lula, e a mesma tendência no governo Dilma, a julgar pelos resultados dos seus primeiros dois anos de gestão. No âmbito federal, os governos do Partido dos Trabalhadores (PT) não diferiram dos governos do Partido da Social Democracia Brasileira (PSDB). 0 perfil dos gastos em saúde não deu conta de atender aos ditames do sonho da reforma sanitária dos anos 1980. Implantado em grande medida do ponto de vista legal, não se materializou na prática. Os números revelam e explicam, em parte, as dificuldades e tensões do SUS (Soares; Santos, 2014).

\section{Os diferentes entendimentos sobre equidade e sua aplicação no SUS}

No Brasil, os esforços para reduzir as iniquidades em saúde consideram a equidade na dotação de recursos financeiros, no acesso a serviços de saúde e nos resultados. Distinguem-se ainda as desigualdades no estado de saúde que expressam desigualdades sociais, de modo que a igualdade no acesso aos serviços de saúde não é suficiente, ainda que necessária, para reduzir as desigualdades no adoecer e no morrer entre os distintos grupos sociais. Essa distinção tem sido considerada importante na formulação de políticas públicas ao se lidar com a noção de necessidades diferentes 
entre os distintos grupos sociais, que são tanto determinadas pela diversidade de sexo, idade e raça, quanto por situações de injustiça social, como renda e acesso a bens e serviços, e ainda por características culturais e subjetivas de grupos e indivíduos (Paim, 20o6).

Paim ressalta que a própria ambiguidade do termo equidade, as diferentes concepções do SUS presentes no processo político de saúde e as diversificadas formas de sua apresentação em municípios e estados brasileiros, mais ou menos próximas aos dispositivos legais e ao projeto da Reforma Sanitária, não permitem afirmar de modo definitivo e peremptório que o SUS seja uma política pública de promoção de equidade. Ou seja, o SUS, enquanto política pública, foi formulado na perspectiva do acesso universal, da igualdade e da justiça social no que diz respeito às necessidades de saúde da população brasileira. $\mathrm{Na}$ medida em que a equidade seja concebida na perspectiva ética e da justiça, essa política pública tem um grande potencial de alcançá-la, não obstante as históricas iniquidades presentes na sociedade brasileira, agravadas pelas novas versões do capitalismo em tempos de globalização (Paim, 2006).

Outros autores, estudando o pensamento de gestores municipais do SUS, ainda que num universo bastante limitado (Granja; Zoboli; Fracolli, 2013), mostram que o sentido de equidade se concentra em duas vertentes: (a) tratar todos de forma igualitária, preponderando a ideia da igualdade e da universalidade do acesso, e (b) tratar os desiguais de forma desigual, assertiva desdobrada em duas outras: (b1) priorizando os mais necessitados, segundo a condição socioeconômica e (b2): priorizando grupos específicos, segundo critérios de risco. No enfoque que prioriza os mais necessitados sob o ponto de vista econômico, clara está a preocupação com a vulnerabilidade apresentada pelos grupos desfavorecidos, que não apenas sofrem maior incidência de doenças, como também uma maior carga de condições crônicas e de incapacidades em idade precoce, o que está em sintonia com as afirmações de Whitehead, no texto que produziu para a OMS em 1990 (Luiz, 2005).

Por outro lado, no aspecto que prioriza grupos específicos, clara está à busca por critérios obje- tivos que auxiliem na definição da alocação de recursos em saúde, segundo risco de adoecimento de grupos específicos. Tal pensamento baseia-se, sobretudo, em critérios de objetividade clínica e epidemiológica. Por outro lado, o utilitarismo, corrente de pensamento bastante difundida entre os planejadores do setor da saúde, prega que o correto, o justo, é a alocação de recursos que possam proporcionar maior benefício e mais saúde para o maior número possível de pessoas (Lucchese, 2003). Entretanto, há que se criticar tal pensamento pela possibilidade potencial de gerar práticas injustas ou discriminatórias contra grupos minoritários (Granja; Zoboli; Fracolli, 2013).

Outros autores brasileiros distinguem duas formas de se analisar a equidade, a que chamam de vertical e horizontal (Travassos; Castro, 2012). A primeira é entendida como desigualdade entre desiguais, ou seja, uma regra de distribuição desigual para indivíduos que se encontram em situações distintas; a segunda corresponde à igualdade entre iguais, ou seja, uma regra de distribuição igualitária entre aqueles que estão em igualdade de condições. Pode-se dizer que as normas que regem o SUS incorporam a definição de equidade horizontal, ou seja, acesso, utilização e tratamento igual para necessidades iguais.

Lembra Escorel que, em geral, o princípio da equidade tem sido operacionalizado em duas principais dimensões: condições de saúde e acesso e utilização dos serviços de saúde. No âmbito das condições de saúde é analisada a distribuição dos riscos de adoecer e morrer em grupos populacionais. Embora variações biológicas como sexo e idade determinem diferenças de morbidade e mortalidade, a maior parte das condições de saúde é socialmente determinada e não decorre de variações naturais ou de livres escolhas pessoais por estilos de vida mais ou menos saudáveis. Os pobres, grupo social e economicamente vulnerável, pagam o maior tributo em termos de saúde, acumulando a carga de maior frequência de distribuição de doenças, sejam de origem infecciosa, crônico-degenerativas ou ainda originadas por causas externas (Escorel, 2008). 


\section{Outros elementos para o debate}

No campo prático, percebe-se que as diferentes correntes de pensamento não dão a homogeneidade necessária à compreensão de como a noção de equidade - e de qual equidade se está falando - deve ser aplicada sistematicamente no âmbito do SUS e das políticas públicas que o integram.

A equidade, como já dito, comporta distintas dimensões em sua análise, dentre as quais são mais comumente utilizadas a oferta (recursos humanos e capacidade instalada); o acesso e utilização de serviços; o financiamento (despesa pública e familiar); a qualidade da atenção; a situação de Saúde e as condições de vida da população.

Assim, acima de tudo, é preciso considerar a equidade como sendo a estratégia para se atingir a igualdade de oportunidades no tocante ao usufruto de boas condições de saúde e de acesso aos serviços de promoção, prevenção e recuperação, levando-se em conta as disparidades de ordem social, étnica, econômica e cultural. Ela poderá ser mensurada através da análise das políticas públicas, no setor da saúde, voltadas para o enfrentamento de tais situações (Nunes et al., 2001).

Cabe, pois, avançar nessa discussão de modo a se estabelecer balizas seguras que resultem na prática de promoção da equidade necessária à concretização do direito à saúde, capazes ainda de produzir, como fruto, a melhoria objetiva de indicadores de saúde, da percepção individual de satisfação para com seu próprio estado de saúde, de familiares e da comunidade a que se pertence. Além disso, que possa ser atingido o ideal de um sistema de saúde que seja capaz de garantir o necessário a todos, levando-se em conta singularidades e necessidades.

\section{Referências}

ALMEIDA, C. Equidade e reforma setorial na América Latina: um debate necessário. Cadernos de Saúde Pública, Rio de Janeiro, v. 18, p. 23-26, 2002. Disponível em <http://dx.doi.org/10.159o/ So102-311X2002000700004>. Acesso em: 12 jun. 2014.

ARENDT, H. A condição humana. Rio de Janeiro: Forense Universitária, 1987.
BAGRICHEVSKY, M. et al. Desigualdades sociais em saúde e práticas corporais: um exercício singular de análise. Saúde e Sociedade, São Paulo, v. 22, n. 2, p. 497-510, 2013.

BRASIL. Ministério da Saúde. Declaração de Alma-Ata. In:____. As cartas da promoção da saúde. Brasília, DF, 2002. Série B: Textos Básicos em Saúde. Disponível em: <http://bvsms.saude. gov.br/bvs/publicacoes/cartas_promocao.pdf〉. Acesso em :10 set. 2014.

BRASIL. Ministério da Saúde. Documento orientador para os debates. In: CONFERÊNCIA NACIONAL DE SAÚDE, 14., 2011, Brasília. Documento... Brasília, DF, 2011. Disponível em: <http://conselho.saude.gov.br/14cns/doc_orientador. html>. Acesso em: 10 set. 2014.

BREILH, J. Las tres 'S' de la determinación de la vida: 10 tesis hacia una visión crítica de la determinación social de la vida y la salud. In: NOGUEIRA, R. P. (Org.). Determinação social da saúde e reforma sanitária. Rio de Janeiro: Centro Brasileiro de Estudos de Saúde, 2010. p. 87-125.

CAMPOS, G. W. S. Reflexões temáticas sobre equidade em saúde: o caso do SUS. Saúde e Sociedade, São Paulo, v. 15, n. 2, p. 23-33, 2006. CARVALHO, G. Financiamento da saúde pública no Brasil no pós-constitucional de 88. Tempus Actas de Saúde Coletiva, Brasília, v. 2, n. 2, p. 3951, jul./dez., 2008.

\section{CONSELHO NACIONAL DE SECRETÁRIOS DE} SAÚDE. O financiamento da saúde. Brasília, DF, 2011.

ESCOREL, S. Os dilemas da equidade em saúde: aspectos conceituais. Brasília: OPAS, 2001.

Disponível em: <http://biblioteca.planejamento. gov.br/biblioteca-tematica-1/textos/saudeepidemias-xcampanhas-dados-descobertas/ texto-83-2013-os-dilemas-da-equidade-emsaude-aspectos-conceituais.pdf $>$. Acesso em: 4 jun. 2014.

ESCOREL, S. Equidade em saúde. In: PEREIRA, I. B.; LIMA, J. C. F. Dicionário da educação profissional em saúde. 2. ed. Rio de Janeiro: Escola 
Politécnica em Saúde Joaquim Venâncio, 2008. Disponível em: <http://www.epsjv.fiocruz.br/ dicionario/verbetes/equsau.html>. Acesso em: 12 jun. 2014.

FLEURY, S. Direitos sociais e restrições

financeiras: escolhas trágicas sobre universalização. Ciência \& Saúde Coletiva, Rio de Janeiro, v. 16, n. 6, p. 2686-2688, jun., 2011.

FLEURY, S.; OUVERNEY A. M. Política de Saúde: uma política social. In: GIOVANELLA, L. et al. (Org.). Políticas e Sistema de Saúde no Brasil. Rio de Janeiro: Fiocruz, 2008. p. 23-64.

GARRAFA, V.; OSELKA, G.; DINIZ, D. Saúde pública, bioética e equidade. Revista de Bioética, Brasília, v. 5, n. 1, p. 1-6, 2009. Disponível em: <http://revistabioetica.cfm.org.br/index.php/ revista_bioetica/article/view/361/462>. Acesso em: 4 jun. 2014.

GOMES, F. B. C. Impasses no financiamento da saúde no Brasil: da constituinte à regulamentação da emenda 29/oo. Saúde em Debate, Rio de Janeiro, v. 38, n. 100, p. 6-17, 2014.

GRANJA, G. F.; ZOBOLI, E. L. C. P.; FRACOLLI, L. A. $O$ discurso dos gestores sobre a equidade: um desafio para o SUS. Ciência \& Saúde Coletiva, Rio de Janeiro, v. 18, n. 12, p. 3759-3764, 2013.

JILLSON, I. A.; EISENSTEIN, E. Direitos à saúde e questões éticas durante a adolescência: desafios atuais! Adolescência \& Saúde, Rio de Janeiro, v. 10, p. 53-6o, maio, 2013. Suplemento 2.

LUCCHESE, P. T. R. Equidade na gestão descentralizada do SUS: desafios para a redução de desigualdades em saúde. Ciência \& Saúde Coletiva, Rio de Janeiro, v. 8, n. 2, p. 439-448, 2003.

LUIZ, O. C. Direitos e equidade: princípios éticos para a saúde. Arquivos Médicos do $A B C$, Santo André, v. 3o, n. 2, p. 69-75, 2005.

MENDES, E. V. 25 anos do Sistema Único de Saúde: resultados e desafios. Estudos Avançados, São Paulo, v. 27, n. 78, p. 27-34, 2013.

NUNES, A. et al. Medindo as desigualdades em saúde no Brasil: uma proposta de monitoramento. Brasília, DF: Organização Pan-Americana da
Saúde, Instituto de Pesquisa Econômica Aplicada, 2001.

OLIVEIRA, C. de. Gastos públicos com saúde cresceram de 2007 a 2009, aponta IBGE.

Disponível em: <http://www.redebrasilatual.com. $\mathrm{br} / \mathrm{saude} / 2012 / \mathrm{ol} /$ gastos-publicos-com-bens-eservicos-de-saude-cresceram-entre-2007-e-2009aponta-ibge>. Acesso em: 21 jan. 2016.

ONU - ORGANIZAÇÃO DAS NAÇÕES UNIDAS. Comitê de Direitos Econômicos, Sociais e Culturais. General Comment No. 14: The Right to the Highest Attainable Standard of Health (Art. 12 of the Covenant). [S. l.]: [s. n.], 200o. Disponível em: <http://www.refworld.org/docid/4538838do. html>. Acesso em: 4 jun. 2014.

PAIM, J. S. Equidade e reforma em sistemas de serviços de saúde: o caso do SUS. Saúde e Sociedade, São Paulo, v. 15, n .2, p. 34-46, 2006.

PINHEIRO, M. C.; WESTPHAL, M. F.; AKERMAN, M. Equidade em saúde nos relatórios das conferências nacionais de saúde pós-Constituição Federal brasileira de 1988. Cadernos de Saúde Pública, Rio de Janeiro, v. 21, n. 2, p. 449-458, 2005.

PIOLA, S. F. Saúde no Brasil: algumas questões sobre o Sistema Único de Saúde (SUS). Brasília: CEPAL, 2009. Texto para Discussão, Instituto de Pesquisa Econômica Aplicada (IPEA).

PIOLA S. F. et al. Financiamento público da saúde: uma história à procura de rumo. Texto para discussão. Rio de Janeiro: Instituto de Pesquisa Aplicada, 2013. Disponível em: <http://repositorio. ipea.gov.br/bitstream/11058/1580/1/TD_1846. pdf >. Acesso em: 12 jun. 2014.

SEN, A. Por qué la equidade en salud? Revista Panamericana de Salud Publica, Washington, DC, v. 11, n. 5-6, p. 302-309, 2002.

SILVA, J. B. da; BARROS M. B. A. Epidemiologia e desigualdade: notas sobre a teoria e a história. Revista Panamericana de Salud Publica, Washington, DC, v. 12, n. 6, p. 375, 2002.

SOARES, A.; SANTOS, N. R. Financiamento do Sistema Único de Saúde nos governos FHC, Lula e Dilma. Saúde em Debate, Rio de Janeiro, v. 38, n. 100, p. 18-25, 2014. 
STARFIELD, B. Improving equity in health: a research agenda. International Journal of Health Services, Westport, v. 31, n. 3, p. 545- 566, 2001.

TRAVASSOS, C.; CASTRO, M.S. M. Determinantes e desigualdades sociais no acesso e utilização dos serviços de saúde. In: GIOVANELLA, L. et al. (Org.). Políticas e Sistema de Saúde no Brasil. Rio de Janeiro: Fiocruz, 2012. p. 183-206.

VIEIRA-DA-SILVA, L. M.; ALMEIDA FILHO, N. Equidade em saúde: uma análise crítica de conceitos. Cadernos de Saúde Pública, Rio de Janeiro, v. 25, p. S217-S226, 2009. Suplemento 2. Disponível em < http://www.scielo.br/scielo. php?pid=So102-311X2009001400004\&script $=$ sci $_{-}$ arttext>. Acesso em: 22 jul. 2015.

WHITEHEAD, M. The concepts and principles of equity and health. International Journal of Health Services, Westport, v. 22, n. 3, p. 429-445, 1992.

\section{Contribuição dos autores}

Barros e Sousa contribuíram com a revisão da literatura e a redação final do artigo. 